\title{
LUNG CANCER DISEASE ANALYZES USING PSO BASED FUZZY LOGIC SYSTEM
}

\author{
P. Bhaktavastalam ${ }^{1}$, S. Narayana Reddy ${ }^{2}$ \\ ${ }^{1}$ Research Scholar in S.V University, ${ }^{2}$ Professor in S.V University \\ ${ }^{1,2}$ Dept. of Electronics \& Communication Engineering. S.V. University, Tirupati, Andhra Pradesh, India.
}

\begin{abstract}
Main objective of this paper to improve accuracy of lung cancer disease investigation utilizing molecule swarm enhancement (PSO) in combination with fuzzy expert system. This paper briefly a introduce fuzzy expert systems and this proposed scheme compared with related methods. Experimental results of the proposed system simulated by MATLAB 2014.
\end{abstract}

\section{INTRODUCTION}

The main objective of optimization is to find the well-suited result to a crisis under a given collection of constraints. Many researchers over the decades have arisen with dissimilar results to linear and non-linear optimization harms. Mathematically optimization obscurity engrosses a fitness function describing the issue, under an arrangement of limitations symbolizing the result space for the problem. Regrettably, most of the Conventional optimization practices are revolved around assessing the first subordinates to put the optima on a given compelled surface. Because of the troubles in assessing the first subordinates, to find the optima for numerous uneven and irregular optimization surfaces, in modern times, more than a few derivatives at no cost optimization algorithms have emerged. The streamlining issue, now-a-days, is characterized as a clever investigate difficulty, where single or multiple agents are utilized to determine the optima on a pursuit scene, speaking to the confined surface for the advancement difficulty.

In 1990s Beernaert and Kennedy enunciate an another answer to the difficult non-linear optimization problem through emulate the communal actions of bird flock, particle the boids system for Craig Reynolds and socio-perception and call their thought the particle swarm optimization in the region of the same time, cost and Storm took a grim challenge to put back the traditional intersect and change operator in GA through alternative operator, and as a result come up with a appropriate discrepancy worker to switch the issue. They planned a new calculation based under this administrator, and called it level of contrast advancement. Together calculations needn't bother with any rising data of the errand to be advanced utilize just ancient arithmetical administrators alongside are hypothetically simple. They can be actualizing inside any processor dialect to a great degree effectively and require minimum parameter change Algorithm presentation does not deteriorate barbarously among the improvement of the search for space greatness too. This issue perchance has a gigantic part in the notoriety of the calculations contained by the space of machine astuteness and artificial intelligence.
The significant parameters of the accepted PSO imitation are $\omega, \mathrm{C} 1, \mathrm{C} 2$, Vmax notwithstanding the swarm size $\mathrm{S}$. The settings of these parameters decide how it improves the inquiry space. Case in point, one can apply a general setting that gives sensible results on most issues, yet from time to time is extremely ideal. Subsequent to the same parameter settings not in any way ensure accomplishment in diverse issues, we should have information of the impacts of the distinctive settings, such that we can pick a suitable setting from issue to issue.

Fuzzy logic be similar to human reasoning in it's utilize of inaccurate data to make assessments. Different conventional logic which necessitates a profound recognizing of a method, exact mathematical statements, and exact numeric qualities, fluffy rationale incorporates a possibility for mindset, which allows chiseling multifaceted plans using an unrivaled level of deliberation starting from our data and ability. Fluffy rationale grants communicating this information with subjective thoughts, for example, amazingly enormous and a protracted time which are mapped into precise numeric decisions. Since data can be explained in a more characteristic by utilizing fluffy sets, numerous choice (and building) damages can be truly streamlined. Fluffy rationale offers a finding morphology that allows genuinely precise human thinking capabilities to be connected to certainties based frameworks.

The theory of fluffy rationale displays a scientific intensity to detain the second thoughts related with human subjective procedures, for example, thinking and thinking. The straight attract close to truths delineation needs the methods for representation the significance of fluffy ideas. As an outcome, draw close got from first request rationale don't given suitable applied structure for managing the portrayal of judicious data, given that such realities is by its character both lexically inaccurate and no downright. The improvement of fluffy rationale was persuaded in substantial decide by the require for an applied structure which can address the issue of lexical ambiguity. 
The principle of this paper is to notice the phase of cancer and to perform so; we used the prevalent and significant signs as the most powerful signs in finding of period of lung cancer. We also utilized, the period of signs being in progress as one of the most vital factors in the exposure of this illness, which was never employed before in previous associated papers.

\section{FUZZY LOGIC}

It is troubles explain oversee framework strategy that fit execution in framework range from simple minimal implanted microcontrollers to cumbersome system multichannel PC or work place based information getting hold of and control frameworks. It can be executing in equipment, programming, or a gathering of together. It gives a simple strategy to show up at an accurate end based winning indistinguishable, vague, erroneous, strident, or truant data. In this way to deal with control issues mirror how somebody would settle on choice just much faster.

It fuse simple, guideline based IF $\mathrm{X}$ alongside $\mathrm{Y}$ after that $\mathrm{Z}$ moves toward to tackle sort out trouble to some degree than endeavor to demonstrate an association precisely. This model is Empirically-based, depending on administrator learning very than their innovative tolerating of the plan. It needs some numerical parameter in order to enact for instance what is measured vital mix-up and imperative rateof-progress of-blunder, aside from right standards of these data are ordinarily not basic if not exceptionally open execution is essential in which case experiential change would choose them, for example, a straightforward warmth control framework can utilize a just hotness input sensor whose information is subtract from the power sign to ascertain "mistake" and afterward time-separate to give way the misstep review or rate-of-progress of-mistake, after this call blunder spot. Mistake quality have unit of degs $\mathrm{F}$ and a little blunder measured to be $2 \mathrm{~F}$ though a major mistake is $5 \mathrm{~F}$. The blunder spot might then have unit of degs/min with a little mistake dab being $5 \mathrm{~F} / \mathrm{min}$ and a huge one being $15 \mathrm{~F} / \mathrm{min}$. These standards don't need to be customary and can be "changing" on one event the framework is in administration so as to streamline presentation. As a rule, FL is sympathetic to the point that the plan will in all likelihood work the most punctual time excluding any alteration.

\section{EXISTING SYSTEM}

This revises offered a fuzzy rule-based specialist scheme used for scenario of the breast cancer in well and injurious females. The exits FIS predict the danger of the breast cancer rising features linked to scientific account of females. The fuzzy specialist system was effectively developed and knowledgeable for assess the danger of breast tumor in 80 female dataset with or without breast cancer who referred to sickbay for breast cancer selection.

The presentation outcome reveals an average exactness of $95 \%$ for all categories in the dataset (healthy and unhealthy). This approach provides wide spread of information to help the physician in reaching a more logical conclusion for a more accurate prediction of the breast cancer hazard. In addition, it can categorize females by means of high risk of mounting breast cancer.

This approach is helpful for early on identification of the breast cancer and correct healing at early on stage of the mounting breast cancer, which can develop the continued existence rate. Our expectations effort is to discover the FIS by means of malleable compute technique such as ANFIS (Adaptive Neural Network Fuzzy Inference System) and GA (Genetic Algorithm) and confirm the FIS outcome on additional genuine datasets.

\section{PROPOSED SYSTEM}

In this paper introduces the advance particle swarm optimization with fuzzy expert system for analysis of lung cancer. The membership function and calculated input fuzzy sets and their importance discuss with a medical research scholar of lung cancer and moreover a patient profile has been utilized. At lost performance of the proposed PSO based lung cancer analysis system on the information obtained from multiple patients calculated. the outcome of simulation result and comparison with other existing methods indicates the superiority of this proposed scheme to improve the accuracy of the system, PSO in combination with fuzzy system can be employed.

\section{SIMULATION RESULT}

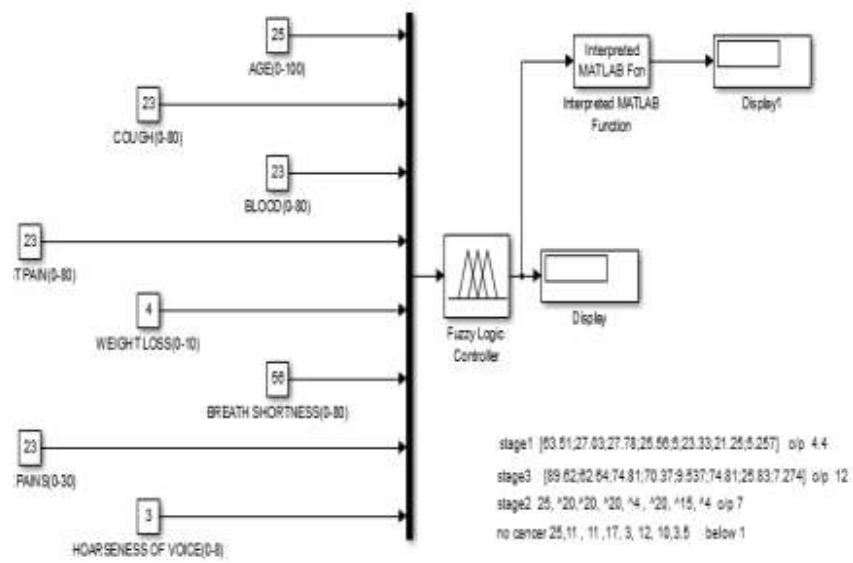

Fig.1. Simulation model

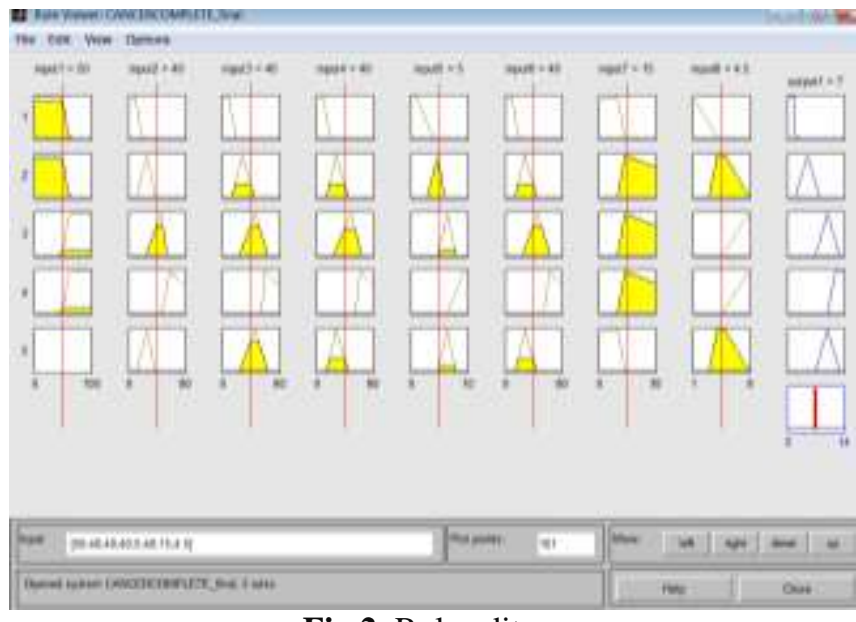

Fig.2. Rule editor 


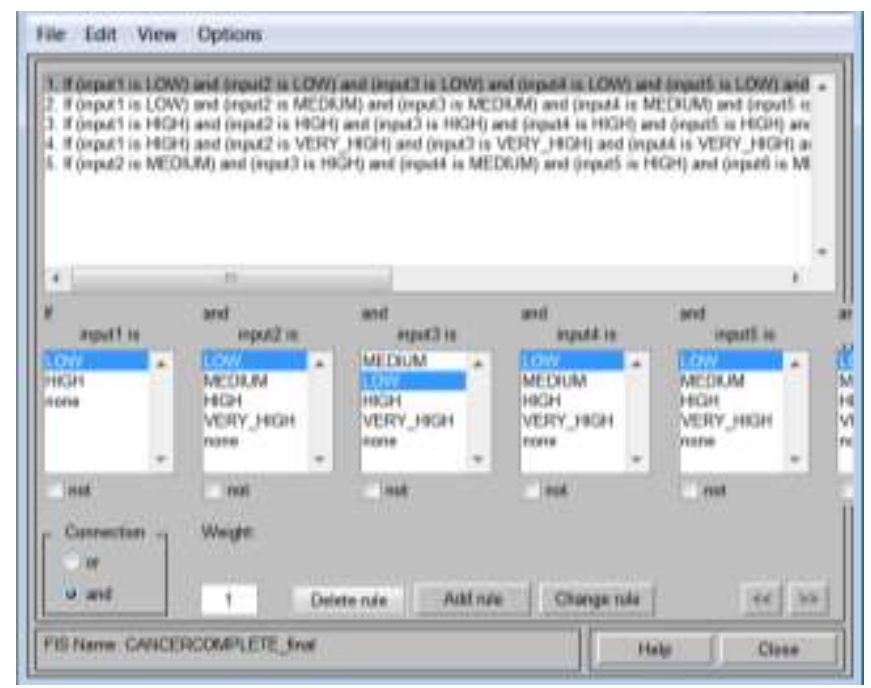

Fig.3. Fuzzy rules

The accuracy of classification methods in lung cancer:

Table-I

\begin{tabular}{|l|l|l|l|}
\hline $\begin{array}{l}\text { Method } \\
\text { Code name }\end{array}$ & $\begin{array}{l}\text { Analyzing } \\
\text { the stage of } \\
\text { cancer\% }\end{array}$ & $\begin{array}{l}\text { Cancer } \\
\text { detecting-no } \\
\text { staging }\end{array}$ & $\begin{array}{l}\text { Average } \\
\text { results } \\
\%\end{array}$ \\
\hline BSH & 82.25 & 87.09 & 84.67 \\
\hline EFR2 & 65.38 & 73.07 & 69.22 \\
\hline FSL1 & 79.03 & 88.70 & 83.86 \\
\hline PSO & 82.30 & 88.80 & 84.26 \\
\hline
\end{tabular}

\section{CONCLUSION}

In this proposed system, we clarified the design and construction of PSO with fuzzy proficient method for the analysis of lung cancer. For calculating the membership functions and inputs and their significance we checked with a medical professionals of lung cancer and also patient's profiles has been employed. Testing results and comparison with further existing schemes point outs the pre-eminence of our proposed scheme, in terms of categorization computational speed and accuracy. But the challenge in designing a scheme is in given that accurate results for detection of lung cancer in premature stages to reduce the difficulty of luxuriant cancer in superior stages. Due to high precision of scheme.

\section{REFRENCES}

[1] Khezri R., Hosseini R., Mazinani M., "A Fuzzy Rulebased Expert System for the Prognosis of the Risk of Development of the Breast Cancer", International Journal of Engineering (IJE) Transaction A: Basics, Vol. 27, No. 10, (October 2014) 1557-1564.

[2] Saleem Dura, M.A and Iyengar, N.Ch.S.N. and Kannan, A., "Enhanced Fuzzy Rule Based Diagnostic Model for Lung Cancer using Priority Values. (IJCSIT)",
International Journal of Computer Science and Information Technologies, Vol. 2 (2011), page no. 707710.

[3] Rahi, M. and Firoozmand, M., "A model based on the fuzzy system to detect lung cancer", First Conference of intelligent computer systems and their applications, Tehran, Payam Noor University, Tehran, (2012).

[4] Phuong, N. H. and Nghi, L. H. and Cho, H. and Kwak, Y. S., "LDDS - A Fuzzy Rule Based Lung Diseases Diagnostic System Combining Positive and Negative Knowledge", National Center for Natural Science and Technology and Kyungpook National University School of Medicine, (2003).

[5] Saleem Durai, M.A. and Iyengar, N.Ch.S.N., "Effective Analyssis and Diagnosis of Lung Cancer Using Fuzzy Rules" International Journal of Engineering Science and Technology, Vol. 2 (2010), page no. 2102-2108. 